\title{
Erratum to "Responsive Inverse Opal Scaffolds with Biomimetic Enrichment Capability for Cell Culture"
}

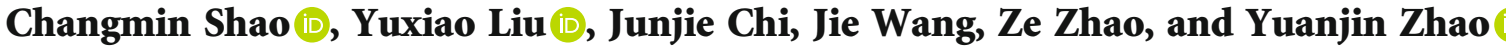 \\ State Key Laboratory of Bioelectronics, School of Biological Science and Medical Engineering, Southeast University, \\ Nanjing 210096, China \\ Correspondence should be addressed to Yuanjin Zhao; yjzhao@seu.edu.cn \\ Received 5 January 2022; Accepted 5 January 2022; Published 4 February 2022 \\ Copyright (C) 2022 Changmin Shao et al. Exclusive Licensee Science and Technology Review Publishing House. Distributed under \\ a Creative Commons Attribution License (CC BY 4.0).
}

In the article titled "Responsive Inverse Opal Scaffolds with Biomimetic Enrichment Capability for Cell Culture" [1], there was an error in Figure 6e. The scale on the $y$-axis was written as $0-100$, whereas it should have been $0-1000$. This error was introduced during the production process, and the publisher apologises for this error. The corrected figure is shown below: 


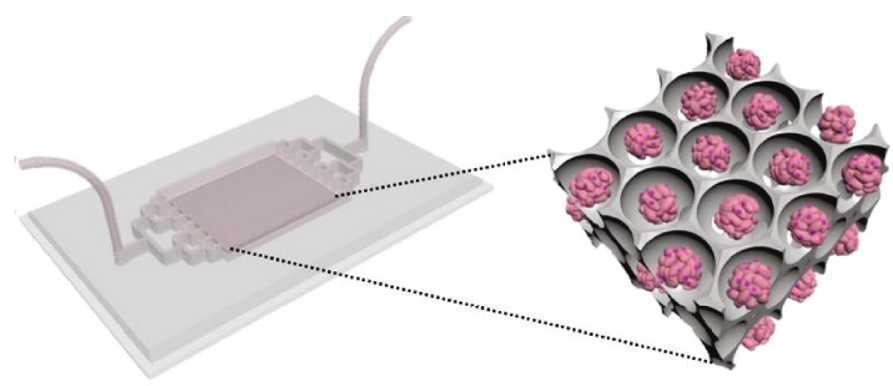

(a)

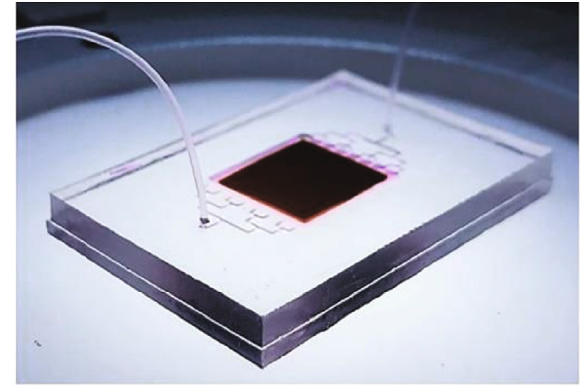

(b)

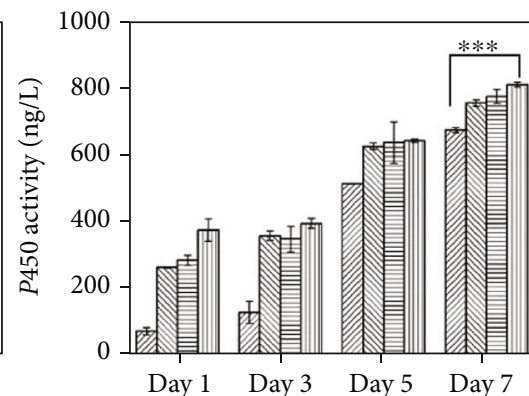

产 HepG2+EC

메미 HepG2+3T3+EC

(d) (e)

FIGURE 6: The applications of the GO hydrogel scaffolds in a liver-on-a-chip system. (a) Schematic of the construction of the liver-on-a-chip. (b) Image of the GO hydrogel scaffold-integrated liver-on-a-chip. (c-e) Albumin secretion (c), urea synthesis (d), and cytochrome P450 expression (e) of HepG2 after coculture with 3T3, ECs, and both 3 T3 and ECs in the liver-on-a-chip system for 7 days; ${ }^{* * *} p<0.01$.

\section{References}

[1] C. Shao, Y. Liu, J. Chi, J. Wang, Z. Zhao, and Y. Zhao, "Responsive Inverse Opal Scaffolds with Biomimetic Enrichment Capability for Cell Culture," Research, vol. 2019, article 9783793, pp. 1-10, 2019. 